\title{
Il Ministero della Salute alle Società medico-scientifiche: garantire l'indipendenza del dibattito scientifico dall'ingerenza delle compagnie del tabacco
}

\author{
Paolo D'Argenio
}

| Ministero della Salute richiama tutti gli Enti del Servizio Sanitario Nazionale, anche convenzionati, università e Società medicoscientifiche a garantire l'indipendenza dalle compagnie del tabacco in sedi di dibattito scientifico.

Nessun tipo di collaborazione è possibile con l'industria del tabacco. Ecco i 7 punti della lettera del Ministero indirizzata alla Federazione Nazionale degli Ordini dei Medici Chirurghi e degli Odontoiatri (FNOMCeO), alla Società Italiana di Tabaccologia (SITAB) e altre Società Scientifiche:

1. Numerosi eventi o convegni organizzati in Italia da Società Scientifiche, Regioni e Ordini professionali, hanno ricevuto contributi finanziari da parte di industrie del tabacco e incluso nei programmi interventi di esponenti delle stesse industrie, a supporto della cosiddetta strategia di "riduzione del danno".

2. Le regole in vigore in Italia vietano il contributo a eventi, attività o persone finalizzato, anche indirettamente, a promuovere il tabacco.

3. Nel 2008, I'Italia ha ratificato un trattato internazionale, la Convenzione quadro per il controllo del tabacco, che vincola il nostro Paese a:

a) Proteggere le politiche dall'influenza dagli interessi dell'industria del tabacco (Articolo 5.3); b) Vietare pubblicità, promozione e sponsorizzazione del tabacco (Articolo 13). Per sponsorizzazione bisogna intendere: qualsiasi forma di contributo pubblico o privato ad un evento, un'attività o una persona che abbia lo scopo o l'effetto, diretto o indiretto, di promuovere un prodotto del tabacco (Articolo 1 del D.Lgs. n. 300/2004).

4. L'industria del tabacco ha immesso sul mercato nuovi prodotti a tabacco riscaldato (IOOS, Glo, Ploom) con una strategia di vendita basata sul concetto di "riduzione del rischio", una falsità visto che essi possono determinare dipendenza da nicotina, indurre gli ex fumatori a ricadere nella dipendenza e gli adolescenti ad acquisirla.

5. L'Istituto Superiore di Sanità ha valutato le IOOS su istanza della Philip Morris e non ha potuto riconoscere il potenziale di riduzione del rischio.

6. In base alle conoscenze attuali, I'approccio della riduzione del rischio o del danno non è una strategia di salute pubblica, ma una strategia di vendita. La strategia di salute mira invece alla disassuefazione dal fumo e dalI'utilizzo di prodotti del tabacco o a base di nicotina.

7. Le strategie dell'industria del tabacco mirano al mantenimento di una vasta platea di consuma- tori, mentre il Ministero è impegnato nello sforzo di difendere la preminenza della salute rispetto agli altri interessi coinvolti nel tabacco che possono stare a cuore delle altre Amministrazioni coinvolte: Ministero dell'Economia, dello Sviluppo Economico, Politiche Agricole e Agenzia dei Monopoli.

[Tabaccologia 2021; XIX(1):44-47] https://doi.org/10.53127/tblg-2021-A008

\section{Paolo D'Argenio}

Editor di tobaccoendgame.it

Associazione Italiana di Epidemiologia (AIE)

Disclosure: I'autore dichiara I'assenza di conflitto d'interessi. 
Agli Assessori alla Sanità delle Regioni e Province Autonome tramite pec

Alla Federazione Nazionale degli Ordini dei Medici Chirurghi e degli Odontoiatri

(FNOMCEO) segreteria@pec.fnomceo.it

Alla Società Italiana di Medicina Generale (SIMG) simg@pec.it

Alla Società Scientifica Interdisciplinare e di Medicina di Famiglia e di Comunità (ASSIMEFAC)

Via Livorno, 36 - 00162 Roma segreteria@assimefac.it

Alla Società Nazionale di Aggiornamento per il Medico e di Medicina Generale

(SNAMID)

Via Matteo Civitali 73 - 20148 Milano segreteria@snamid.it

Alla Società Italiana Medicina dell'Adolescenza (SIMA) c/o Dip. Materno Infantile -

Reparto Pediatria

Ospedale San Raffaele

Università Vita - Salute pozzobon.gabriella@hsr.it

Alla Società Italiana di Tabaccologia

(SITAB)

via G. Scalia 39, 00136 Roma segreteria@tabaccologia.it

OGGETTO: Problematiche relative all'organizzazione di eventi scientifici con il coinvolgimento delle Industrie del tabacco 
Il consumo di tabacco continua a provocare nel mondo 8 milioni di morti all'anno (più di 93 mila solo in Italia). Per contrastare l'epidemia del tabacco, l'Organizzazione Mondiale della Sanità ha adottato nel 2003 la Convenzione Quadro sul controllo del tabacco (FCTC-WHO), ratificata in Italia con la legge n.75 del 2008, che impegna i Paesi ad adottare misure efficaci per ridurre la mortalità e le malattie correlate al consumo di tabacco. L'articolo 5.3 della citata Convenzione prevede che, visto l'inconciliabile conflitto tra gli interessi dell'industria del tabacco e quelli della salute pubblica nella definizione delle politiche di contrasto al tabagismo, gli Stati devono fare in modo che tali politiche non siano influenzate dagli interessi commerciali e di altro tipo dell'industria del tabacco. L'articolo 13, inoltre, prevede il divieto di qualsiasi forma di pubblicità, promozione e sponsorizzazione del tabacco.

Il Decreto legislativo 16 dicembre 2004, n. 300 di attuazione della direttiva 2003/33/CE in materia di pubblicità e di sponsorizzazione dei prodotti del tabacco, all'articolo 1 definisce sponsorizzazione "qualsiasi forma di contributo pubblico o privato ad un evento, un'attività o una persona che abbia lo scopo o l'effetto, diretto o indiretto, di promuovere un prodotto del tabacco".

Occorre ricordare, inoltre, che l'introduzione di nuovi prodotti, quali sigarette elettroniche e prodotti a tabacco riscaldato (Heated Tobacco Products HTP), ha determinato un ampliamento dell'offerta sul mercato e una nuova strategia di vendita che tende a promuovere il concetto di "riduzione del rischio" che deriverebbe dal consumo di tali prodotti.

Evidenze di letteratura indicano che i dispositivi che rilasciano nicotina, come le sigarette elettroniche e i prodotti a tabacco riscaldato, possono determinare dipendenza da nicotina come $\mathrm{i}$ prodotti tradizionali (Liu et al., 2019A; 2019B).

Per gli ex fumatori che, oggi, sono in molti paesi più numerosi dei fumatori, questi dispositivi rappresentano un rischio di ricadere nella dipendenza alla nicotina (Liu et al., 2019B). Preoccupanti sono anche i dati sui consumi degli adolescenti italiani, riportati dalla Global Youth Tobacco Survey, che mostrano come nel 2014 il 23\% dei ragazzi 11-15 anni fumava abitualmente sigarette e 1'8\% utilizzava abitualmente sigarette elettroniche, ma nel 2018 il 21\% fumava sigarette e il 18\% utilizzava sigarette elettroniche, con aumento del numero di adolescenti che vengono a contatto con la nicotina.

Di contro, è recentemente emerso quanto i prodotti in parola non costituiscano in alcun modo un'alternativa valida e sicura al consumo tradizionale di tabacco, stante la presenza, oltre alla nicotina, di agenti cancerogeni e sostanze tossiche e nocive, nonché il verificarsi di eventi letali per patologie polmonari conseguenti all'uso di sigarette elettroniche, ad oggi segnalati negli Stati Uniti e in Gran Bretagna.

Nel nostro Paese, inoltre, la valutazione effettuata secondo la normativa vigente dall'Istituto Superiore di Sanità su un prodotto di tabacco riscaldato, su istanza dell'Azienda produttrice (Philip Morris) ha evidenziato che non sia possibile, allo stato attuale e sulla base della documentazione fornita dal proponente, riconoscere la riduzione delle sostanze tossiche, né di stabilire il potenziale di riduzione del rischio del prodotto in esame rispetto ai prodotti da combustione, a parità di condizioni di utilizzo.

L'approccio della riduzione del rischio o del danno, pertanto, allo stato attuale delle evidenze, non può essere adottato quale strategia di salute pubblica, che mira invece alla disassuefazione dal fumo e dall'utilizzo di prodotti del tabacco o contenenti nicotina.

Tale posizione è in linea con quanto ad oggi sostenuto dall'OMS,attraverso le decisioni adottate nell'ambito della Convenzione Quadro, e dall'Unione Europea che ha dedicato specifici articoli della direttiva 40/2014/EU alla regolamentazione delle sigarette elettroniche e dei prodotti di nuova generazione. 
A settembre 2017, Philip Morris International, una delle più grandi compagnie di tabacco del mondo, ha creato la "Foundation for a Smoke-Free World" (Fondazione per un mondo senza fumo), con una dotazione di quasi 1 miliardo di dollari, in 12 anni. La Fondazione sostiene di essere un organismo scientifico indipendente per accelerare la fine del fumo. Degli 80 milioni di dollari ricevuti da Philip Morris International nel 2018, la Fondazione ha speso 6,5 milioni in assegni di ricerca, 7,6 milioni in attività di comunicazione, per la maggior parte svolte da organizzazioni di pubbliche relazioni e 7,03 milioni in spese per il personale. 47,5 milioni di dollari non sono stati ancora spesi.

Occorre pertanto ribadire che mentre le strategie poste in atto dall'Industria del tabacco mirano al mantenimento di una vasta platea di consumatori, le strategie di salute pubblica non possono che essere finalizzate alla progressiva diminuzione dei consumi di tutti i prodotti del tabacco, al calo della prevalenza dei fumatori e alla conseguente riduzione delle gravissime patologie fumo correlate. Il Ministero della salute è impegnato nell'arduo compito di "advocacy" verso le altre Amministrazioni coinvolte, per la promozione di azioni intersettoriali e misure efficaci che seguano le indicazioni della Convenzione OMS, affinché la pluralità di interessi correlati ai prodotti del tabacco non prevalgano sul supremo interesse della tutela della salute.

Premesso quanto sopra, è necessario evidenziare che, recentemente, numerosi eventi o convegni organizzati in Italia da alcune associazioni o Società scientifiche, promossi da Istituzioni regionali e da Ordini professionali, hanno ricevuto contributi finanziari da parte di industrie del tabacco e hanno incluso nei programmi scientifici interventi da parte di esponenti delle stesse industrie, a supporto della cosiddetta strategia di "riduzione del danno", che anche nell'ambito di un rapporto individuale medico-paziente non può essere ritenuta un valido approccio clinico, essendo disponibili approcci terapeutici anche farmacologici di provata efficacia.

Nel riaffermare, pertanto, che l'unica strategia perseguibile, in un'ottica di salute pubblica, è la disassuefazione totale dal fumo e dal consumo di altri prodotti del tabacco, le scriventi Direzioni, acquisito il parere favorevole del vertice politico di questo Ministero,richiedono la collaborazione di codeste Istituzioni ed Enti raccomandando di vigilare affinché tutti gli enti del SSN, anche convenzionati, Università, e società mediche e scientifiche si impegnino a garantire l'indipendenza dalle compagnie del tabacco in sedi di dibattito scientifico, e a condurre attività di advocacy affinché aumenti la consapevolezza delle conseguenze dell'ingerenza dell'industria del tabacco e dell'importanza di improntare le scelte preventive e terapeutiche esclusivamente sulla base di consolidate evidenze scientifiche, nell'interesse del singolo individuo e della collettività.

Grato per la preziosa collaborazione istituzionale

Il Direttore Generale della Direzione generale della prevenzione sanitaria

Dr. Giovanni Rezza

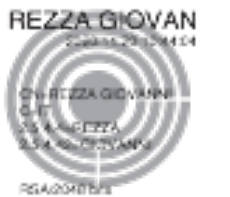

Il Direttore Generale della Direzione generale delle professioni sanitarie e delle risorse umane del Servizio Sanitario Nazionale

Dr.ssa Rossana Ugenti

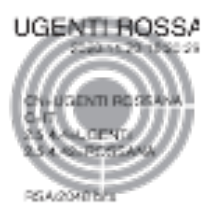

